\title{
Models for the dynamical evolution of the Magellanic System
}

\author{
Kenji Bekki \\ School of Physics, University of New South Wales, Sydney, NSW, 2052, Australia \\ email: bekki@phys.unsw.edu.au
}

\begin{abstract}
I discuss the following five selected topics on formation and evolution of the LMC and the SMC based on fully self-consistent chemodynamical simulations of the Magellanic Clouds (MCs): (1) formation of bifurcated gaseous structures and young stars in the Magellanic bridge (MB), (2) formation of the Magellanic stream (MS) due to the tidal interaction between the LMC, the SMC, and the Galaxy within the last 2 Gyrs, (3) origin of the observed kinematical differences between $\mathrm{H}$ I gas and stars in the SMC, (4) formation of stellar structures dependent on their ages and metallicities in the LMC, and (5) a new common halo model explaining both the latest HST ACS observations on the proper motions of the LMC and the SMC and the presence of the MS in the Galactic halo. I focus exclusively on the latest developments in numerical simulations on formation and evolution of the Magellanic system.
\end{abstract}

Keywords. galaxies: evolution, galaxies: halos, galaxies: interactions, galaxies: kinematics and dynamics, Magellanic Clouds, galaxies: stellar content, dark matter

\section{Introduction}

Tidal interaction between the Magellanic Clouds and the Galaxy has long been considered to play vital roles in different aspects of their evolution, such as the formation of the Magellanic stream and the Magellanic Bridge from the SMC (e.g., Murai \& Fujimoto 1980, Gardiner \& Noguchi 1996, GN; Muller \& Bekki 2007), long-term star formation histories (e.g., Yoshizawa \& Noguchi 2003), chemical evolution (e.g., Hill et al. 2000), formation of a bar and a thick disk in the LMC (e.g., Bekki \& Chiba 2005; BC), and globular cluster formation (Bekki et al. 2004a, b). Previous observational and theoretical studies suggested that hydrodynamical interaction between the gas disk of the LMC and the hot halo gas of the Galaxy can be responsible not only for the formation of the MS (Mastropietro et al. 2005) but also for the recent star formation history of the LMC (de Boer et al. 1998; Mastropietro, these proceedings). Although the past orbits of the MCs are key parameters that can determine the roles of the above tidal and hydrodynamical effects in the Magellanic evolution (BC), they are not so well constrained by observations.

Recent proper motion measurements of the MCs by the Advanced Camera for Surveys (ACS) on the Hubble Space Telescope (HST) have reported that the LMC and the SMC have significantly high Galactic tangential velocities $\left(367 \pm 18 \mathrm{~km} \mathrm{~s}^{-1}\right.$ and $301 \pm 52 \mathrm{~km} \mathrm{~s}^{-1}$, respectively) and thus suggested that the MCs could be unbound from each other (Kallivayalil et al. 2006, K06; Piatek et al. 2008). These new observations have strongly suggested that previous orbital models adopted in the MS formation models (e.g., BC) are not consistent with the observed proper motions and thus that new physical ingredients would need to be included in any dynamical modes of the MCs for self-consistency with the HST results. Recent numerical simulations have demonstrated that the MS models consistent with the $H S T$ proper motion measurements can not explain self-consistently 
the locations of the MS and the leading arms projected onto the sky (Bekki \& Chiba 2008a).

Keeping these observational and theoretical developments in mind, I discuss the latest observational results on structures, kinematics, and star formation histories of the MCs based on the results of numerical simulations of dynamical evolution of the MCs. I particularly focus on (i) stellar and gaseous distributions of the MB (Muller et al. 2003; Harris 2007), (ii) fine structures in the MS (e.g., Brüns et al. 2005), (iii) rotational and non-rotational kinematical in gas and stars of the SMC (Harris \& Zaristky 2006), (iv) structural differences between different stellar populations (van der Marel 2001; Olsen \& Massey 2007), such as young super-giants, AGB stars, and old PNe populations in the LMC (Reid \& Parker 2006), and (v) possible presence of a common dark matter halo surrounding the MCs (Bekki 2008).

\section{The Bridge}

Although the origin of the MB has been suggested to be closely associated with the LMC-SMC interaction about 0.2 Gyr ago (e.g., GN; Yoshizawa \& Noguchi 2003), detailed comparison between the simulated and observed properties of the MB has not been done until quite recently. Key questions as to the MB raised by previous observations include (i) how the tidal interaction model explains both the apparent kpc-scale giant $\mathrm{H}$ i hole in the MB and the bifurcated kinematics in a self-consistent manner (e.g., Muller et al. 2003), (ii) why the metallicities of young stars in the inter-Cloud and the Bridge regions are significantly lower than those $(<[\mathrm{Fe} / \mathrm{H}] \sim-0.6)$ of stars in the SMC (e.g., Rolleston et al. (1999), (iii) why the MB appears to consist almost purely of gas (e.g., Harris 2007), and (iv) how molecular clouds can be formed in a very low density gaseous region of the MB during the last LMC-SMC interaction (e.g., Mizuno et al. 2006). I here discuss these questions based on "the tidal interaction model" in which the MCs interact strongly with each other about 0.2 Gyr ago to form the MB from the gas disk of the SMC. The details of the SMC model are given in Bekki \& Chiba (2007a) and the orbits of the models discussed here are shown in Fig. 1 for initial velocities from GN and K06. Fig. 2 shows

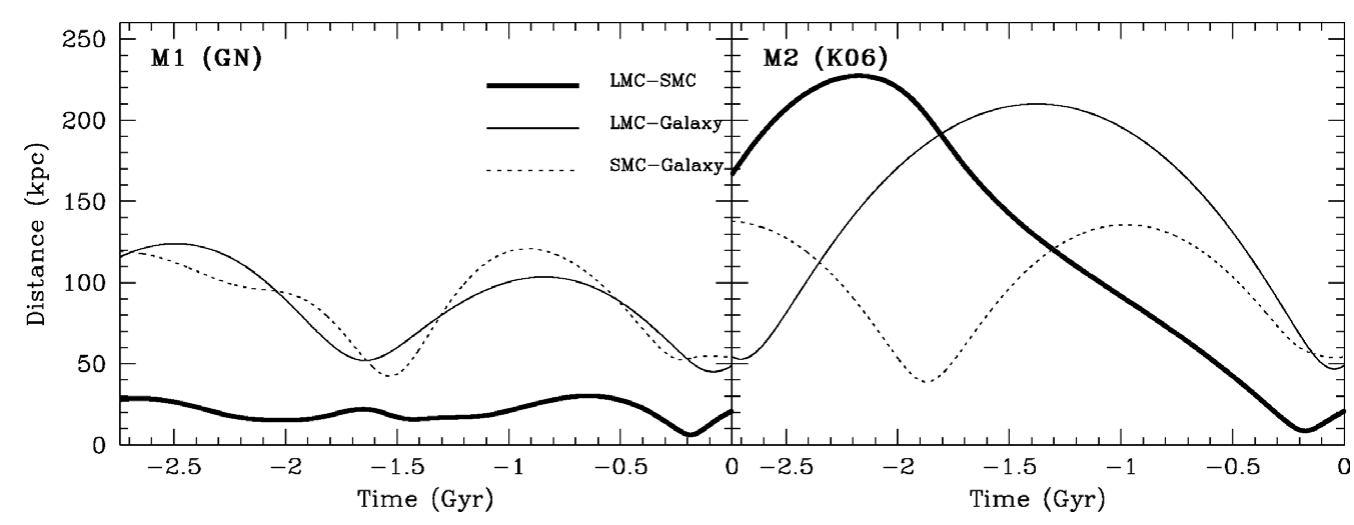

Figure 1. The time evolution of the distances between LMC-SMC (thick solid), LMC-Galaxy (thin solid), and SMC-Galaxy (dotted) for the last 2.7 Gyr in the models M1 (left, referred to as GN) consistent with orbits of MCs by GN and M2 (right, K06) consistent with those by K06. Note that the M1 model can keep the binary status of the MCs whereas the M2 one does not, which suggests that the MS would not be formed from the outer gas disk of the SMC in models with the K06 orbital types. 
an example of the final particle distributions of the models in which both the MS and the MB can be formed from the SMC.

Recent numerical simulations have shown that (i) the $\mathrm{MB}$ is one of two tidal arms (or tails) formed from the outer gas disk of the SMC as a result of the last LMCSMC tidal interaction about 0.2 Gyr ago and (ii) the observed apparent $\mathrm{H}$ I hole (or loop) can be reproduced simply as a projection of the counter-arm (Muller \& Bekki 2007). Chemodynamical numerical simulations (Bekki \& Chiba 2008a) showed that young stars with ages less than 0.1 Gyr formed in the MB during the last interaction can originate from the very outer part of the gas disk in the SMC so that they have very low metallicities $([\mathrm{Fe} / \mathrm{H}] \sim-0.8)$. They also suggested that the metallicity distribution function of the young stars strongly depends on the initial metallicity gradient of gas in the SMC. The latest results of fully self-consistent chemodynamical simulations by Bekki \& Chiba (2008a) have demonstrated that the MB inevitably contains old stars with the surface mass densities of $6-300 \times 10^{4} \mathrm{M}_{\odot} \mathrm{deg}^{-2}$ depending on initial stellar distributions of the modeled SMC. The results of these numerical simulations based on the tidal interaction model are well consistent with observations on physical properties of the MB (Muller et al. 2003) and thus suggest that the origin of the MB can be understood in the context of the last LMC-SMC interaction without invoking energydeposition processes such as supernova events and stellar winds.

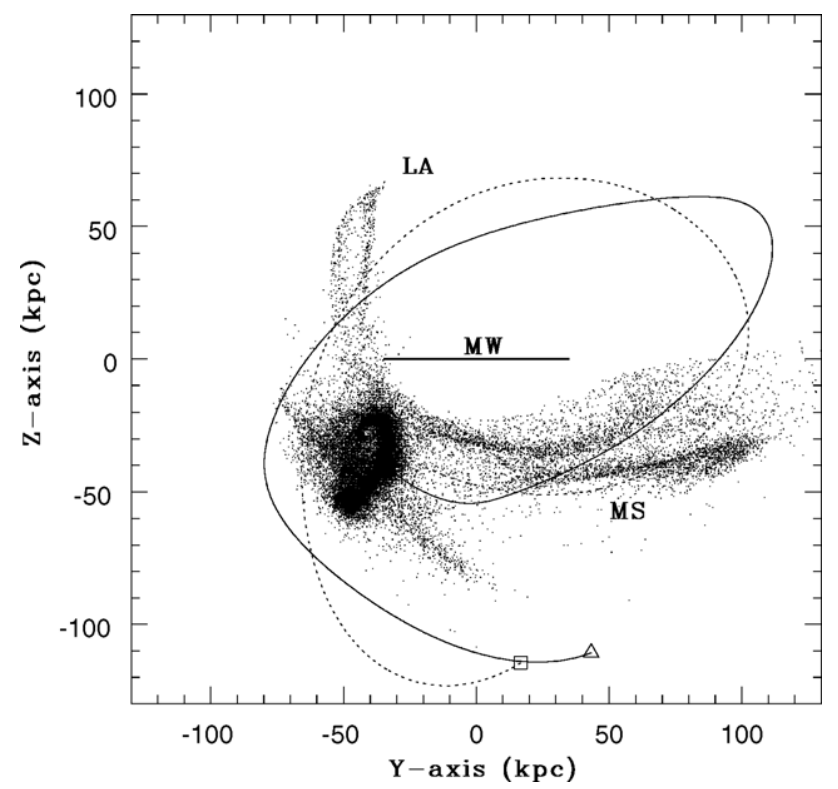

Figure 2. The final distribution of gas of the SMC projected onto the $y$ - $z$ plane for the new "dwarf spheroidal model" (Bekki \& Chiba 2007a; Bekki \& Chiba 2008a) in which the SMC has both a stellar spheroid and an extended H I gas. This is the distribution after the strong tidal interaction between the MCs and the Galaxy around 1.5 Gyr ago and that between the LMC and the SMC around 0.2 Gyr ago. Owing to the interaction, both the MS and the MB can be formed (only the MS can be clearly seen owing to a larger scale of view). The orbits of the LMC (triangle) and the SMC (square) with respect to the center of the Galaxy are shown by solid and dotted lines, respectively. For comparison, the Galaxy with the disk size of $17.5 \mathrm{kpc}$ is shown by a thick solid line. The GN orbital type is adopted here in order to show a successful model in reproducing the formation of the MS, the leading arms, and the MB. The masses of the LMC and the SMC are assumed to be $2 \times 10^{10} \mathrm{M}_{\odot}$ and $3 \times 10^{9} \mathrm{M}_{\odot}$, respectively. 


\section{The Stream}

One of observational evidences that strongly support the tidal interaction model for the MS formation is the presence of the leading arm (e.g., Putman et al. 1998): this can not be simply explained by the ram pressure model in which the MS originates from the LMC. Recent observational studies have confirmed that the MS shows bifurcation in the structure and kinematics (e.g., Brüns et al. 2005) and it also has multiple streams (Stanimirović et al. 2008). These new observational results are consistent with the results of numerical simulations on the MS formation from the past LMC-SMC-Galaxy interaction about 1.5 Gyr ago (Connors et al. 2006). Although previous tidal interaction models are quite successful in explaining a number of fundamental properties of the MS, it has the following three problems: (i) the SMC is modeled as a rotating disk galaxy (e.g., GN), which is inconsistent with observations (e.g., Harris \& Zaritsky 2006), (ii) the total gas mass in the MS and inter-Cloud region appears to be significantly smaller than the observed one (Yoshizawa \& Noguchi 2003) for the model with the mass of the SMC being $3 \times 10^{9} \mathrm{M}_{\odot}$, and (iii) the simulated location of the "kink" in the leading arm around $b=30^{\circ}$ is not consisted with the observed one $\left(b=0^{\circ}\right)$. In order to overcome these problems, we have constructed a new SMC model in which the SMC is represented by a "dwarf spheroidal" with an extended massive H I gas disk. These characteristics in the new SMC models are consistent with the latest observations (e.g., Harris \& Zaristky 2006; Stanimirović et al. 2004).

Fig. 3 shows that the new model with the GN orbital type ("classical" MS model) can explain both the bifurcated structures and no or little stars in the MS in a self-consistent manner. Owing to the initial large gas mass in the extended H I gas disk around the stellar spheroid, the total gas mass of the MS, the MB, and the inter-Cloud regions can be as large as $5 \times 10^{8} \mathrm{M}_{\odot}$ depending on the mass and extension of the $\mathrm{H}$ I disk. These two main streams in the MS originate from two spirals (or tidal arms) formed from the LMC-SMC-Galaxy interaction around 1.5 Gyr ago: it should be noted here that
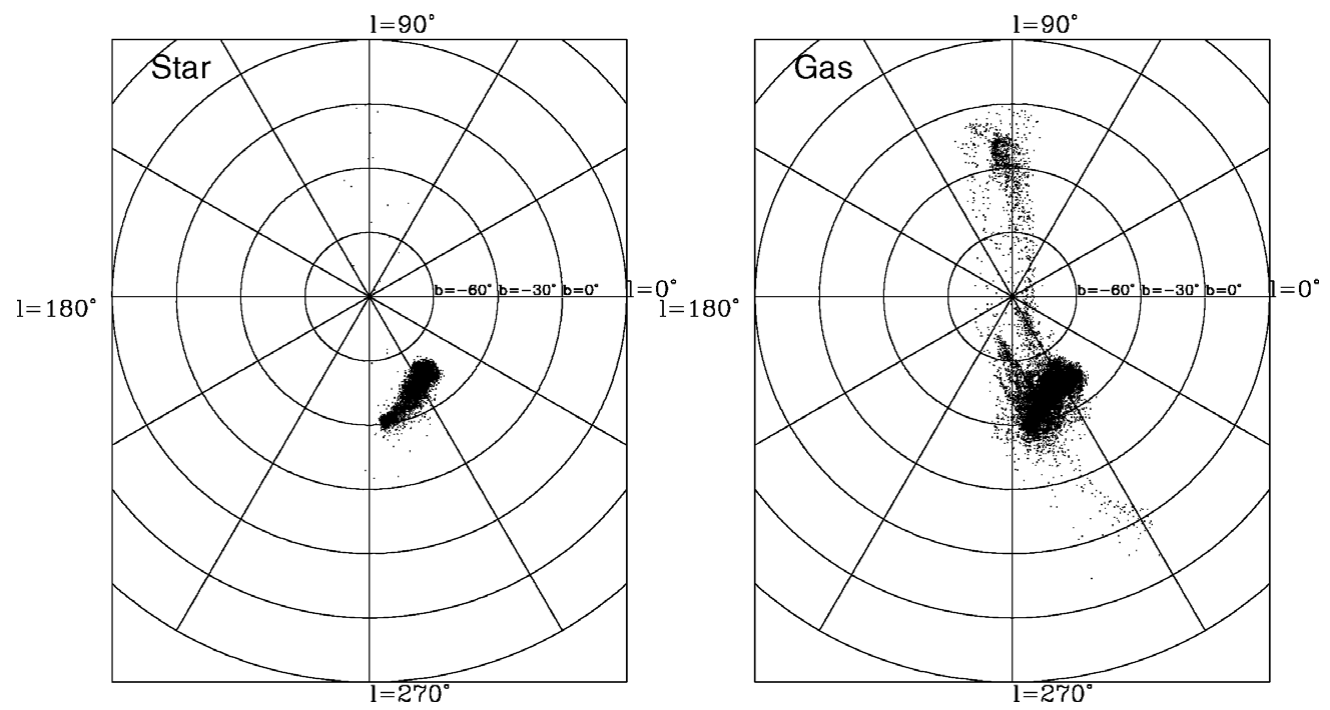

Figure 3. The projected distributions of stars (left) and gas (right) in the new dwarf spheroidal model for the SMC with the total mass of $3 \times 10^{9} \mathrm{M}_{\odot}$ and the gas mass fraction of 2 (i.e., gas mass twice as large as stellar mass). Clearly bifurcation of the MS can be seen in the vicinity of the SMC, and the leading arm also can be well reproduced. Note that the tidal interaction model does not predict the presence of a significant number of stars in the MS. 
the leading arm is also composed of two streams, though this is not clear in the $(l, b)$ projection. The observed $b=0$ kink in the leading arm can not be explained simply by the new models: recent simulations by Bekki et al. (2008), however, have demonstrated that hydrodynamical interaction between the outer gas of the Galaxy and the leading arm can be responsible for the formation of the $b=0$ kink. Our extensive parameter survey for different past orbits of the MCs have led us to conclude that the new models with the K06 orbital types can not explain the locations of the MS and the leading arm at all. This result implies that if the observed proper motions of the MCs are true ones for the centers of mass for the MCs, we would need to consider new physical ingredients in the tidal interaction model for self-consistency.

\section{Kinematical differences between $\mathrm{H}_{\mathrm{I}}$ and older stars in the SMC}

The latest survey of 2046 red giant stars has reported that the older stellar components of the SMC have a velocity dispersion $(\sigma)$ of $\sim 27.5 \mathrm{~km} \mathrm{~s}^{-1}$ and a maximum possible rotation of $\sim 17 \mathrm{~km} \mathrm{~s}^{-1}$ (Harris \& Zaritsky 2006). This result is consistent with other kinematical studies based on radial velocities of other old and intermediate-age stellar populations such as PNe and carbon stars (Russell \& Dopita 1992; Hatzidimitiriou et al. 1993) and thus suggests that the older stellar component is a spheroid that is primarily supported by its velocity dispersion. Recent high-resolution $\mathrm{H}$ I observations have revealed that the SMC has a significant amount of rotation with a circular velocity $\left(V_{\mathrm{c}}\right)$ of $\sim$ $60 \mathrm{~km} \mathrm{~s}^{-1}$ (Stanimirović et al. 2004). These observations on stellar and gaseous kinematics in the SMC suggest that there is a remarkable difference in kinematics between older stellar populations and $\mathrm{H}$ I gas in the SMC.

It would be possible that the rotating gas disk of the SMC can be gradually formed via gas accretion after the formation of the older spheroidal component (Bekki \& Chiba 2008b). As a result of this, directions of intrinsic spin axes of older stars and gas in the SMC could be significantly different to each other, like polar-ring galaxies. In this accretion scenario, there needs to be a fine-tuning of the projected spin directions of stellar and gaseous disks in the SMC for explaining the observed kinematical properties. Although this scenario is not unreasonable, it is unclear why the SMC has a stellar spheroid only for older stellar population in this scenario. Furthermore, it seems unlikely that gas can be accreted gradually onto the SMC until recently, because the tidal field of the Galaxy can strongly suppress the gas accretion onto the SMC from its outer halo.

Recent numerical studies have proposed a new scenario in which the SMC could have experienced a major merger event ("dwarf-dwarf merging") between two gas-rich dwarf irregulars (dIs) long time ago in which both the older stellar spheroid and the rotating gas disk were created (Bekki \& Chiba 2008b). In this scenario, both the stellar spheroid and the rotating $\mathrm{H}$ I disk can be formed almost simultaneously in the last merger event, though the epoch of the merger event can not be specified. Fig. 4 describes how dwarfdwarf merging can transform two dIs into a new dwarf with a central spheroid and an extended gas disk. Owing to strong violent relaxation in the central region of the merger, the inner stellar disks are completely destroyed and form a slightly flattened spheroidal component with a half-mass radius of $2.0 \mathrm{kpc}$. Although the gas disk of the larger dI can be temporarily disturbed strongly by the merging, it finally becomes a new extended gas disk after dissipative merging with that of the smaller dI. The gaseous component shows rotation with the maximum rotational velocity of $59 \mathrm{~km} \mathrm{~s}^{-1}$ and a small central velocity dispersion of $\sigma=24 \mathrm{~km} \mathrm{~s}^{-1}$ (i.e., $V / \sigma \sim 2.5$ ) whereas the stellar one shows a smaller amount of rotation of $V \sim 20 \mathrm{~km} \mathrm{~s}^{-1}$ and a larger maximum velocity dispersion of $\sigma \sim 48 \mathrm{~km} \mathrm{~s}^{-1}$ (i.e., $V / \sigma \sim 0.4$ ). 


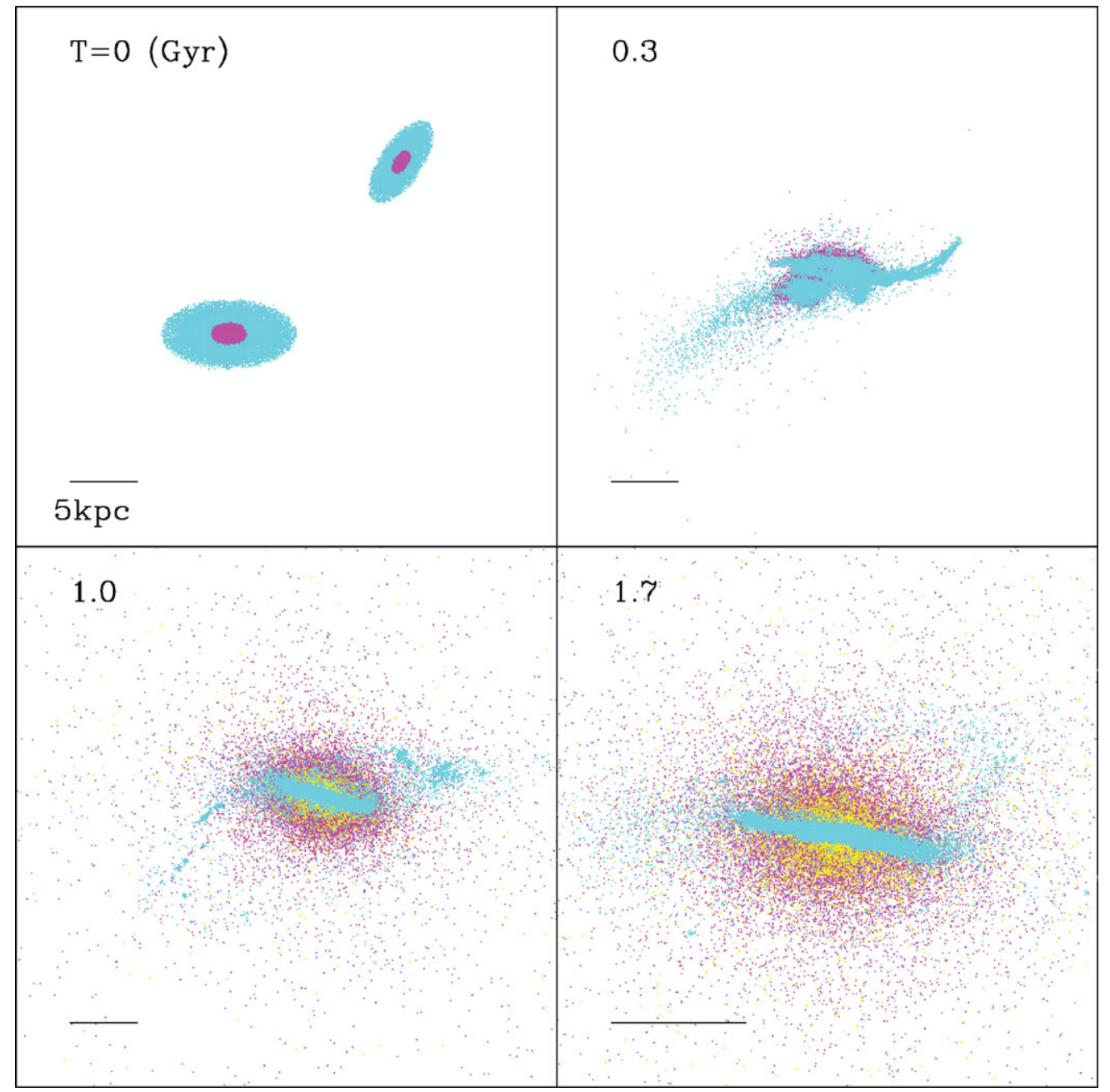

Figure 4. Mass distributions of old stars (magenta), gas (cyan), and new stars (yellow) of the unequal-mass dI-dI merger with the mass ratio of 0.5 projected onto the $x$ - $z$ plane at four different time steps. The time $T$ in units of Gyr is shown in the upper left corner of each panel. Frames measure $40 \mathrm{kpc}$ in the first three and $20 \mathrm{kpc}$ in the last so that the extended disk can be more clearly seen at $T=1.7$ Gyr. Only old stars can be seen for the central $1.25 \mathrm{kpc}$ of two dIs in the first frame, because the stellar particles are overlaid on gaseous ones: gas particles exist in the central $1.25 \mathrm{kpc}$.

Thus the ancient merger scenario can explain well the observed kinematical properties of the SMC and thus suggests that the SMC was previously two dIs. The scenario also provides the following three implications on the origin of the MCs. Firstly, there should be a negative metallicity gradient within the central $2 \mathrm{kpc}(\Delta[\mathrm{Fe} / \mathrm{H}] / \Delta R \sim-0.05$ dex $\mathrm{kpc}^{-1}$ ) for the gaseous component (in the very early history of the SMC) in the sense that the inner part is more metal-rich. The outer part of the remnant $(R>3 \mathrm{kpc})$, which is composed mostly of gas $\left(f_{\mathrm{g}}>0.6\right)$, shows $[\mathrm{Fe} / \mathrm{H}]<-0.95$ owing to severe suppression of star formation and the resultant much less efficient chemical enrichment. These results imply that if the MS can be formed from tidal stripping of gas from the SMC, the metallicity of the MS should be quite low. Secondly, the dI-dI merging leading to the formation of the SMC occurred long before the strong LMC-SMC interaction 
commenced about 3-4 Gyr ago (BC): possibly this merger event might have occurred far away from the Galaxy in order to have the low relative velocity between two merging dIs. We consider that the low relative velocity would be possible, because the two dIs were either initially a pair or in a very small group (of galaxies) with a smaller circular velocity that merged the outer region of the Galaxy's halo long time ago.

Thirdly, there should be a "dip" in the age - metallicity relation (AMR) for stellar populations in the SMC: at the epoch of the last merger event, the mean metallicity of newly born stars can be significantly lower than those formed before the merging. This dip is due to star formation from metal-poor gas transfered from outer gas disks or merger progenitor dIs in the merger scenario: the dip would be possibly seen in the AMR of star clusters in the SMC. If this scenario is correct, a key question is when the SMC experienced such a dwarf-dwarf merger event. Since stellar populations formed before the merger event should have dynamically hot kinematics in this scenario, the youngest age of stellar populations that show both spheroidal distributions and no or little rotation can correspond to the epoch when the merging occurred. Recent observations of AGB stars in the SMC have reported that (i) the average age of the old and intermediate stellar populations is $7-9$ Gyr and (ii) the stars have a more regular distribution and appear to be a slightly flattened ellipsoid (Cioni et al. 2000): the merger event might have occurred about $7-9$ Gyr ago.

\section{Different dynamical properties between different stellar populations in the LMC}

Recent numerical simulations have shown that formation of a stellar bar, a thick stellar disk, and a dynamically hot stellar halo in the LMC is a natural result of the past tidal interaction between the LMC and the Galaxy (BC). Therefore, it is not surprising that the LMC is observed to have the above three dynamical properties: I here do not intend to discuss the origin of these (see BC for more discussion). One of the very intriguing observational results is that there appears to be a significant difference in projected distributions between stellar populations with different ages. H I gas and young stellar populations are observed to show clearly the presence of peculiar spirals arms that are the most likely to be formed from the last LMC-SMC interaction about 0.2 Gyr ago (StaveleySmith et al. 2003; Olsen \& Massey 2007). The intermediate-age stellar populations such as AGB/RGB/carbon stars show no spiral structures but clearly have a off-center bar (van der Marel 2001). The projected distribution of the PNe, which includes possibly very old stellar populations in the stellar halo, shows neither spiral arms nor a bar (e.g., Reid \& Parker 2006), which is remarkably different from those of young and intermediate-age stars (e.g., Olsen \& Massey 2007). It remains unclear why there can be a significant difference in structural properties between stellar populations with different ages in the LMC.

We consider that this structural difference has something to do with different kinematics in stellar populations with different ages. It is likely that the LMC can have an AMR similar to that in the Galaxy in the sense that older stars have higher velocity dispersions. If this is the case, it is an interesting dynamical problem how differently stellar populations having different initial velocity dispersions before the commencement of tidal interaction response to the tidal perturbation. We have constructed a new "twocomponent" disk model of the LMC in which the stellar disk have both cold (i.e., smaller velocity dispersion) and hot (larger) components. We have investigated (i) dynamical responses of the cold component with no bar and the hot one with a bar to tidal perturbation from the SMC about 0.2 Gyr ago and (ii) those of the cold and hot components 

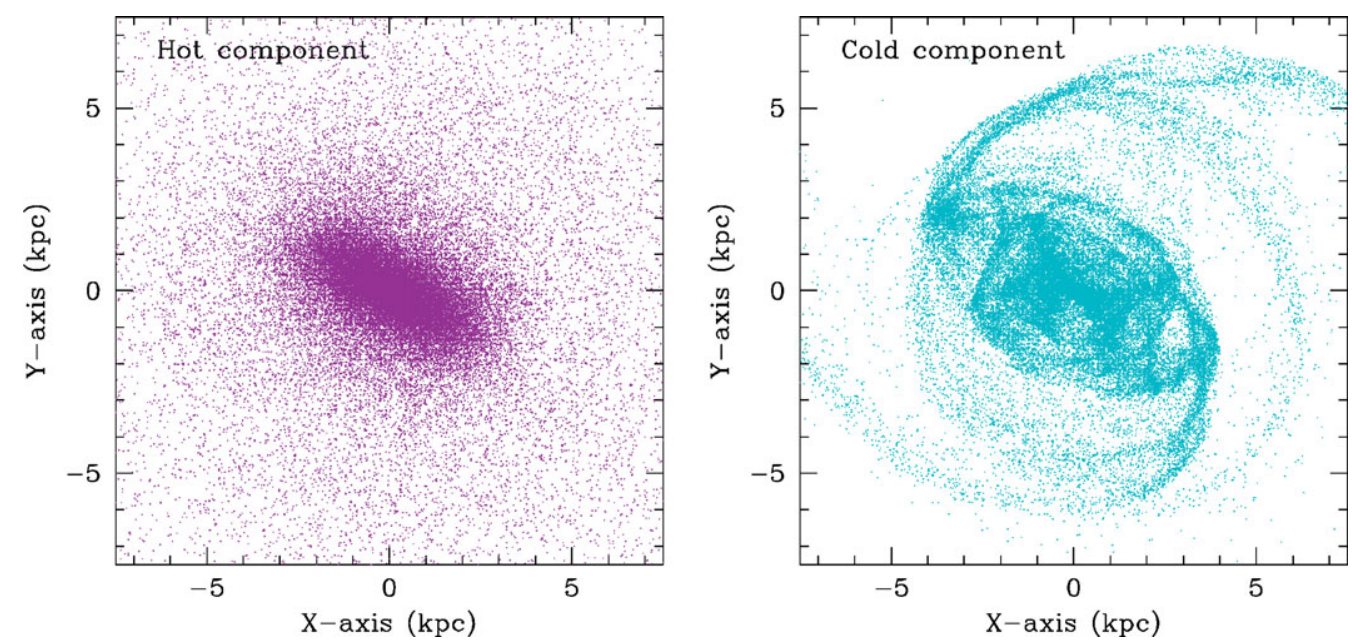

Figure 5. Final distributions of stars in the hot (left) and cold (right) component of the LMC disk interacting with the SMC (after the last $\sim 0.5$ Gyr interaction). The strong LMC-SMC interaction occurs around 0.2 Gyr ago in this model. Note that tidal arms can be seen only in the cold component.
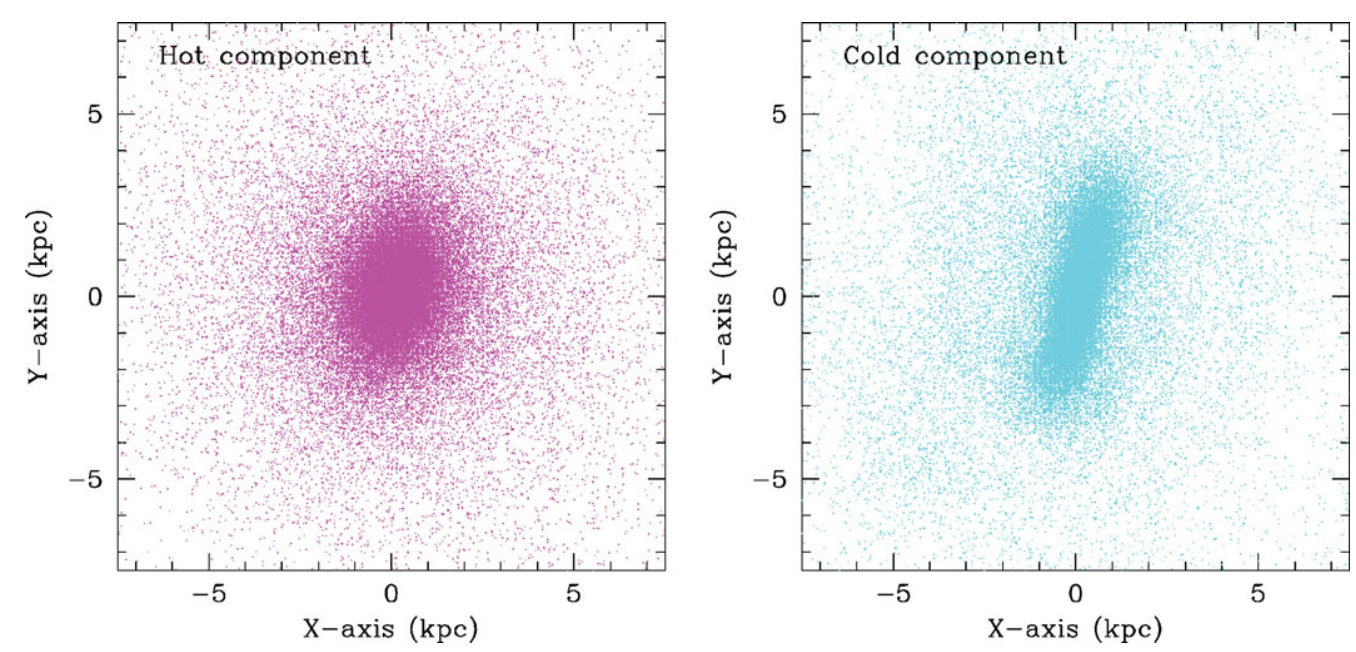

Figure 6. Final distributions of stars in the hot (left) and cold (right) component of the LMC disk interacting with the Galaxy. Note that a strong bar can be seen only in the cold component.

with neither bars nor spiral arms to the tidal perturbation of the Galaxy about 5 Gyr ago.

Fig. 5 shows final distributions of stars in the cold and hot components for the LMC after the LMC-SMC interaction about 0.2 Gyr ago. It is clear that although the cold component shows spiral arms in the central region of the disk, the hot one does not (even though it initially has a bar that can induce spiral arm formation). This is mainly because higher velocity dispersion of stars in the hot component can strongly suppress the formation of spiral arms (or tidal arms) during the LMC-SMC interaction. Fig. 6 shows final distributions of stars in the cold and hot components for the LMC after the LMC-Galaxy interaction about 5 Gyr ago. The hot component does not clearly show a stellar bar whereas the cold one clearly has a strong bar. This is mainly because higher 
velocity dispersion of stars in the hot component can strongly suppress the formation of a bar during the LMC-Galaxy interaction.

These results imply that the absence of spiral arms in intermediate-age stellar populations such as AGB/RGB stars can be understood, if these stars already had a higher velocity dispersion before the LMC-SMC interaction about 0.2 Gyr ago. Equally, the absence of a bar in the present $\mathrm{PNe}$ population can be understood, if their progenitor low-mass stars already had a high velocity dispersion before the LMC-Galaxy interaction about 5 Gyr ago. Thus our simulations have first shown that dynamical responses of stellar populations with different velocity dispersions to tidal perturbation to the SMC and the Galaxy can be different and thus that there can be significant difference in structural properties between stars with different ages. An important observational question related to the above explanations is whether older stars really have higher velocity dispersion in the LMC: it is doubtlessly worthwhile to investigate observationally how stellar kinematics can depend on ages of stars in the LMC.

\section{A possible common halo of the MCs}

Recent cosmological N-body simulations of the pair galaxy formation based on a $\Lambda$ cold dark matter $(\Lambda \mathrm{CDM})$ cosmology have shown that the pair formation like the MCs can occur at $z<0.33$ corresponding to less than 3.7 Gyr ago for a canonical set of cosmological parameters (Ishiyama et al. 2008). Li \& Helmi (2008) have investigated merging histories of subhalos in a Milky Way-like halo using high-resolution simulations based on a $\Lambda$ CDM model and thereby demonstrated that about one-third of the subhalos have been accreted in groups. These results imply that the MCs can have a common dark matter halo that was either formed during the LMC-SMC binary formation or was a previously central part of the much larger Magellanic group which previously had the MCs and other satellite galaxies in the Galaxy. Since previous dynamical models of the MCs do not consider the common halo at all, it is a very interesting problem how the orbits of the MCs can change if they have a common halo.

We have investigated this problem by using the canonical backward integration scheme (Murai \& Fujimoto 1980) for the derivation of the past orbits of the MCs in the models with the mass of the common halo ranging from $10^{10} \mathrm{M}_{\odot}$ to $8 \times 10^{10} \mathrm{M}_{\odot}$ (see the detail of the models in Bekki 2008). In order to demonstrate more clearly the roles of the common halo in keeping the binary status of the MCs, we have also investigated the models without the common halo. It should be stressed here that initial velocities in these models are consistent with those in K06. Fig. 7 shows that the model with the common halo can keep their binary status (i.e., the LMC-SMC distance being less than $50 \mathrm{kpc}$ ) for more than 2 Gyr whereas the model without the common halo can not. These results clearly demonstrate that the common halo can play a role in keeping the binary status of the MCs through its gravitational influence. These thus imply that the MS formation models consistent with K06 can be constructed, if a common halo of the MCs is considered in the models.

If the MCs have a common halo, their evolution can be influenced by the gravitational field of the halo to some extent. One of possible effects of the common halo is that it can shorten the time scale of LMC-SMC merging owing to more efficient dynamical friction. Another effect would be that the gravitational field of the halo can dynamically heat up the disk of the LMC more strongly than the Galaxy does. These possible effects have been already investigated by our N-body simulations on the evolution of the Magellanic group consisting of the MCs and their common halo (yet without other dwarfs) around the Galaxy. The main results of the simulations are briefly summarized as follows. Firstly, it is 


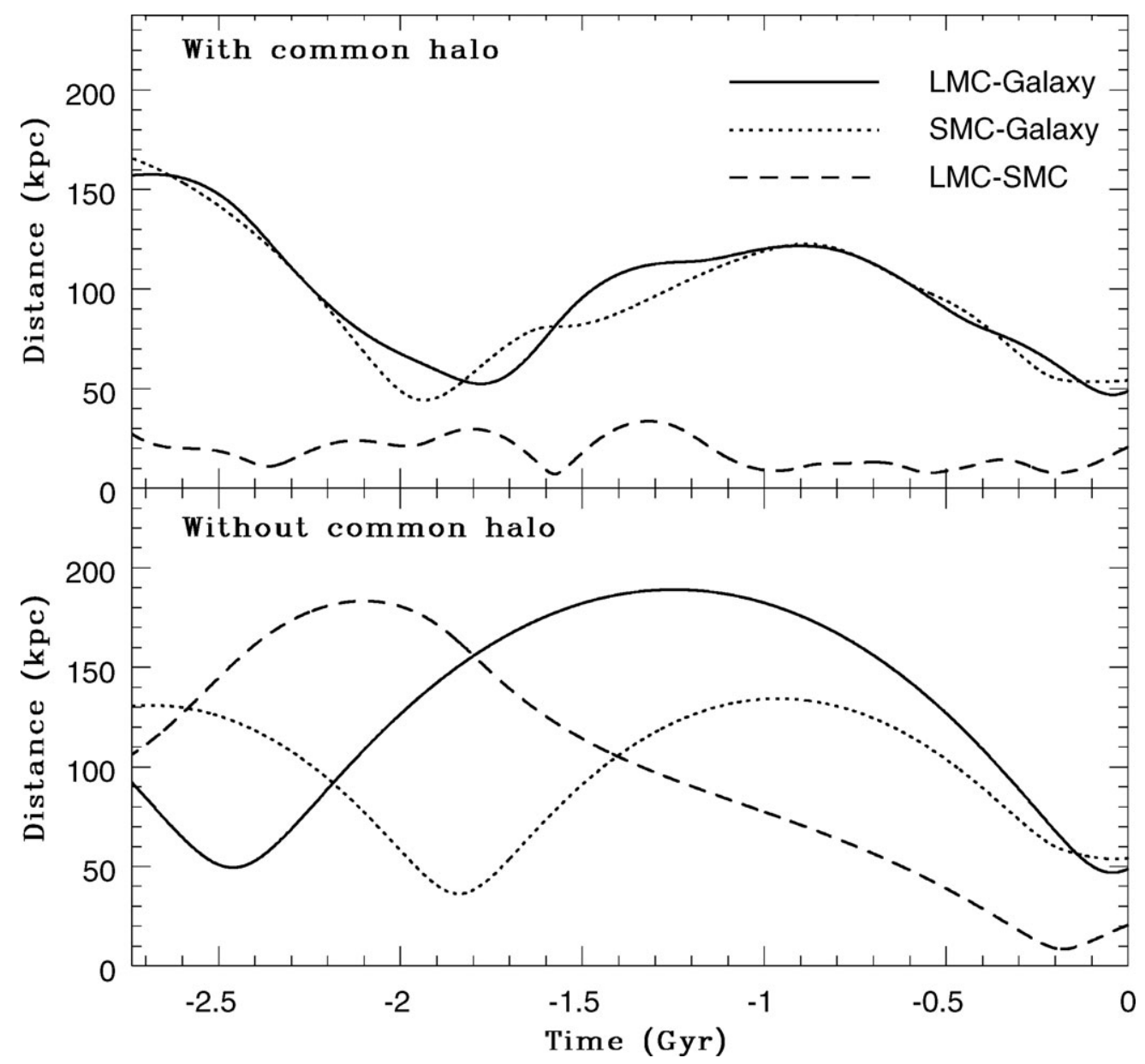

Figure 7. Time evolution of the distances between the LMC and the Galaxy (solid), the SMC and the Galaxy (dotted), and the LMC and the SMC (dashed) for the last $\sim 2.7$ Gyr in the model with (upper) and without (lower) the common halo of the MCs. The time $T=0 \mathrm{Gyr}$ and $T=-2.7$ Gyr mean the present and $2.7 \mathrm{Gyr}$ ago, respectively, in this figure. For these models, the masses of the LMC, the SMC, and the common halo and the scale length of the halo are $M_{\mathrm{L}}=2.0 \times 10^{10} \mathrm{M}_{\odot}, M_{\mathrm{S}}=3.0 \times 10^{9} \mathrm{M}_{\odot}, M_{\mathrm{ch}}=4.0 \times 10^{10} \mathrm{M}_{\odot}$, and $a_{\mathrm{ch}}=10 \mathrm{kpc}$, respectively. Note that the MCs can keep their binary status for the last 2.7 Gyr only for the model with the common halo.

possible for us to construct a model with a massive common halo in which the LMC-SMC do not merge within 2 Gyr owing to tidal heating of the group by the Galaxy. Secondly, stellar tidal streams can be formed as a result of interaction between the LMC and the common halo for most models: this would be a problem if there are no observational evidences for the presence of tidal streams from the LMC. Thirdly, the LMC can show a higher degree of lopsidedness owing to the tidal field of the common halo.

\section{Summary: future works}

Recent chemodynamical simulations significantly improved their predictive power of the dynamical models of the MCs and thereby addressed a number of problems that had not been discussed at all until recently: bifurcated structures in the MB and the MS, 
chemical abundances of the MCs and the inter-Cloud regions, long-term star formation histories of the MCs, origin of thick disks and bars in the LMC, and stellar and gaseous kinematics of the SMC. Although these numerical simulations have explained a number of fundamental observations of the MCs (e.g., the MS), they have not been so successful in reproducing some of important observations. For example, the observed off-center bar in the LMC has not been well reproduced yet, though the last LMC-SMC interaction is demonstrated to play a role in forming the off-center bar (Bekki \& Chiba 2007b). Also, the rotating kinematics of the old globular cluster in the LMC, which is in a striking contrast with the dynamically hot stellar halo, has not been reproduced by simulations (Bekki 2007). The models for hydrodynamical interaction between the Galactic halo gas and the MCs (Mastropietro et al. 2005) needs to be significantly improved, because such interaction can be potentially important for the formation of fine structures in the MS and the leading arms. Formation of star clusters, which are fundamental building blocks of the $M C s$, needs to be investigated by high-resolution galaxy-scale simulations which enable us to discuss the importance of the Magellanic environments in the formation of star clusters from giant molecular clouds (Hurley \& Bekki 2008). It seems to me that currently the most realistic dynamical model of the Magellanic system is not so self-consistent as to explain variously different observational properties of the MCs simultaneously: a lot of work is ahead of us.

\section{References}

Bekki, K. 2007, MNRAS, 380, 1669

Bekki, K. 2008, ApJ, 684, L87

Bekki, K., Beasley, M. A., Forbes, D. A., \& Couch, W. J. 2004a, ApJ, 602, 730

Bekki, K., Couch, W. J., Beasley, M. A., Forbes, D. A., Chiba, M., \& Da Costa, G. 2004b, ApJ, 610, L93

Bekki, K. \& Chiba, M. 2005, MNRAS, 356, 680 (BC)

Bekki, K. \& Chiba, M. 2007a, MNRAS, 381, L11

Bekki, K. \& Chiba, M. 2007b, PASA, 24, 21

Bekki, K. \& Chiba, M. 2008a, PASA, submitted

Bekki, K. \& Chiba, M. 2008b, ApJ, 679, L89

Bekki, K., Chiba, M., \& McClure-Griffiths, N.M. 2008, ApJ, 672, L17

Brüns, C., Kerp, J., Staveley-Smith, L., et al. 2005, A\&SA, 432, 45

Cioni, M. -R. L., Habing, H. J., \& Israel, F. P. 2000, A\&A A, 359, L9

Connors, T. W., Kawata, D., \& Gibson, B. K. 2006, MNRAS, 371, 108

de Boer, K. S., Braun, J. M., Vallenari, A., \& Mebold, U. 1998, A\& A, 329, L49

Gardiner, L.T. \& Noguchi, M., 1996, MNRAS, 278, 191 (GN)

Harris, J. 2007, ApJ, 658, 345

Harris, J. \& Zaritsky, D. 2006, AJ, 131, 2514

Hatzidimitriou, D., Croke, B. F., Morgan, D. H., \& Cannon, R. D. 1997, A\& $A S$, 122, 507

Hill, V., Francois, P., Spite, M., Primas, F., \& Spite, F. 2000, A\&A, 364, L19

Hurley, J. R. \& Bekki, K. 2008, MNRAS, 389, L61

Ishiyama, T. et al. 2008, in B. Koribalski \& H. Jerjen (eds.), Galaxies in the Local Volume, Astrophysics and Space Science Proceedings), in press

Kallivayalil, N., van der Marel, R. P., \& Alcock, C. 2006, ApJ, 652, 1213

Li, Y. \& Helmi, A. 2008, MNRAS, 385, 1365

Mastropietro, C., Moore, B., Mayer, L., Wadsley, J., \& Stadel, J. 2005, MNRAS, 363, 509

Mizuno, N., Muller, E., Maeda, H., et al. 2006, ApJ, 643, L107

Muller, E. \& Bekki, K. 2007, MNRAS, 381, L11

Muller, E., Stanimirović, S., Zealey, W., \& Staveley-Smith, L., 2003, MNRAS, 339, 105

Murai, T. \& Fujimoto, M. 1980, PASJ, 32, 581 
Olsen, K. A. G. \& Massey, P. 2007, ApJ, 656, L61

Piatek, S., Pryor, C., \& Olszewski, E. W. 2008, AJ, 135, 1024

Putman, M. E., Gibson, B. K., Staveley-Smith, L., et al. 1998, Nature, 394, 752

Reid, W. A. \& Parker, Q.A. 2006, MNRAS, 365, 401

Rolleston, W. R. J., Dufton, P. L., McErlean, N. D., \& Venn, K. A. 1999, A\& $A, 348,728$

Russell, S. C. \& Dopita, M. A. 1992, MNRAS, ApJ, 384, 508

Stanimirović, S., Staveley-Smith, L., \& Jones, P. A. 2004, ApJ, 604, 176

Stanimirović, S., Hoffman, S., Heiles, C., et al. 2008, ApJ, 680, 276

Staveley-Smith, L., Kim, S., Calabretta, M. R., Haynes, R. F., \& Kesteven, M. J. 2003, MNRAS, 339,87

van der Marel, R. P. 2001, AJ, 122, 1827

Yoshizawa, A. \& Noguchi, M. 2003, MNRAS, 2003, 339, 1135

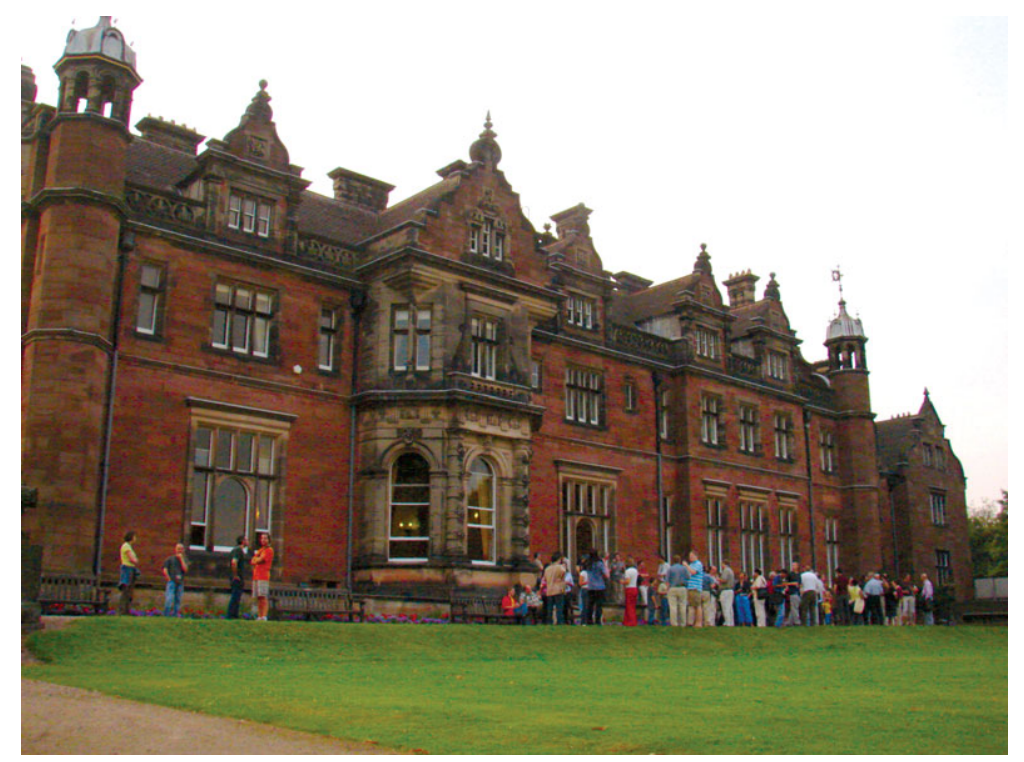

Keele Hall, with dusk setting in. 